\title{
Agave azul y el desarrollo sustentable en la cuenca baja del río Ayuquila, Costa Sur de Jalisco (1994-2004)
}

\author{
Peter R.W. Gerritsen* \\ Jesús Juan Rosales Adame* \\ Arturo Moreno Hernández ${ }^{* *}$ \\ Luis Manuel Martínez Rivera*
}

Resumen: ${ }^{1}$ El agave azul (Agave tequilana Weber) es un cultivo nuevo para la cuenca del río Ayuquila en la Costa Sur de Jalisco, y es importante para su progreso agropecuario. Sin embargo, se observan efectos socioambientales negativos. Desde 2003 se han estudiado las condiciones del cultivo y su expansión, así como alternativas posibles. Los resultados muestran un crecimiento acelerado desde su introducción, a mitad de la década de 1990. Se destaca una concentración de propietarios privados y a través de contratos. Si bien sembrar esta planta puede ser redituable, las tendencias cíclicas de sobreproducción y escasez han originado una falta de incentivos para hacerlo y un deterioro de la capacidad productiva. Se discuten las perspectivas del avance agropecuario basado en el cultivo del agave azul, y

\footnotetext{
* Instituto Manantlán de Ecología y Conservación de la Biodiversidad (IMECBIO), Centro Universitario de la Costa Sur (CUCSUR), Universidad de Guadalajara. Avenida Independencia Nacional \#151, C. P. 48900, Autlán de Navarro, Jalisco, México.

Correo electrónico: petergerritsen@cucsur.udg.mx

** Departamento de Producción Agrícola, cucsur, Universidad de Guadalajara. Avenida Independencia Nacional \#151, C. P. 48900, Autlán de Navarro, Jalisco, México.

${ }^{1}$ El proyecto general recibió financiamiento de la Secretaría de Educación Pública, cucsur y del Swiss National Centre of Competence in Research (NCCR) North-South: Research Partnerships for Mitigating Syndromes of Global Change.
} 
se ofrecen algunas recomendaciones para fomentar la sustentabilidad de su sistema de producción comercial.

Palabras clave: Agave tequilana, occidente de México, Ayuquila, tequila, dinámica regional, globalización.

Abstract: The blue agave crop (Agave tequilana Weber) is a new crop for the Ayuquila watershed in the Southern part of the state of Jalisco in Western Mexico. It has become an important crop in regional agricultural development. However, it has also had a negative social and environmental impact. Since 2003, a research project was implemented in order to analyze the regional conditions of blue agave expansion and production, as well as the identification of sustainable alternatives. Even though blue agave can be a profitable crop, the cyclic tendencies of overproduction and scarcity have caused a lack of incentives for its production and a permanent degradation of the regional productive capacity. The article concludes with some practical recommendations, as well as a discussion of perspectives on sustainable development based on blue agave production.

Key words: Agave tequilana, Southern México, Ayuquila, tequila, regional development, globalization.

\section{Introducción}

En la actualidad se reconocen los efectos negativos del modelo neoliberal promovido por el Estado mexicano sobre el campo que ha perjudicado la sustentabilidad rural; además, está relacionado en forma directa con el proceso de globalización (Morales 2004; Waters 1995). Muchos agricultores no se han beneficiado del neoliberalismo y la globalización (Toledo 2000); como consecuencia, en el campo mexicano hay problemas de desarticulación de las economías campesinas, emigración hacia las zonas urbanas y a Esta- 
dos Unidos y pérdida de conocimientos y tradiciones productivas, entre otros. En ese sentido, las políticas agropecuarias de México impiden la integración de la mayoría de los productores pequeños en el proceso de modernización del agro, además muchas de ellas han resultado contradictorias (Morales 2004).

El cultivo del agave azul es un ejemplo de agricultura comercial, relacionado con el mercado global. Mientras el tequila permanezca en el gusto de los consumidores nacionales y extranjeros seguirá expandiéndose, en las regiones de la Denominación de Origen del Tequila (D.о.т.) e incluso fuera de ella. Es interesante analizar el sistema de producción del agave azul, ya que evoluciona en relación con su demanda; en las últimas décadas se ha intensificado el uso de insumos externos (fertilización, control de plagas y malezas), así como el incremento de plantas por superficie; también se trabaja ya con más tecnología. Las técnicas nuevas de producción difieren del enfoque sustentable, pues al cambiar los sistemas productivos también han cambiado, de manera desfavorable, las relaciones sociales de producción, y en el mismo sentido los efectos al medio ambiente, que anteponen ante todo la ganancia económica.

En la cuenca baja del río Ayuquila, al sur de Jalisco (véase figura 1), el área de interés aquí, el agave azul es un cultivo nuevo surgido en 1994 a consecuencia de la expansión de áreas de siembra nuevas de compañías tequileras, acompañadas con un paquete tecnológico preestablecido que se fomenta sobre todo a través de la agricultura de contrato adoptada por los campesinos como inversión, debido al éxito económico que tuvo de 1999 a 2002, derivado de la escasez de agave (Bowen et al. 2004; Nava et al. 2007; Gerritsen y Martínez 2010).

En este artículo se describen diferentes aspectos de la expansión del cultivo de agave azul en la cuenca baja del río Ayuquila. El objetivo central es mostrar una imagen de la magnitud de este cultivo, en términos cuantitativos, enfocándose en los factores que la causaron. Asimismo, se busca entender — de manera preliminar- las transformaciones que ha generado en los sistemas productivos locales. A continuación se presentan primero algunas nociones teóricas y el diseño del estudio, después los resultados, y para terminar una discusión y conclusión. 


\section{Figura 1}

Ubicación de los municipios estudiados

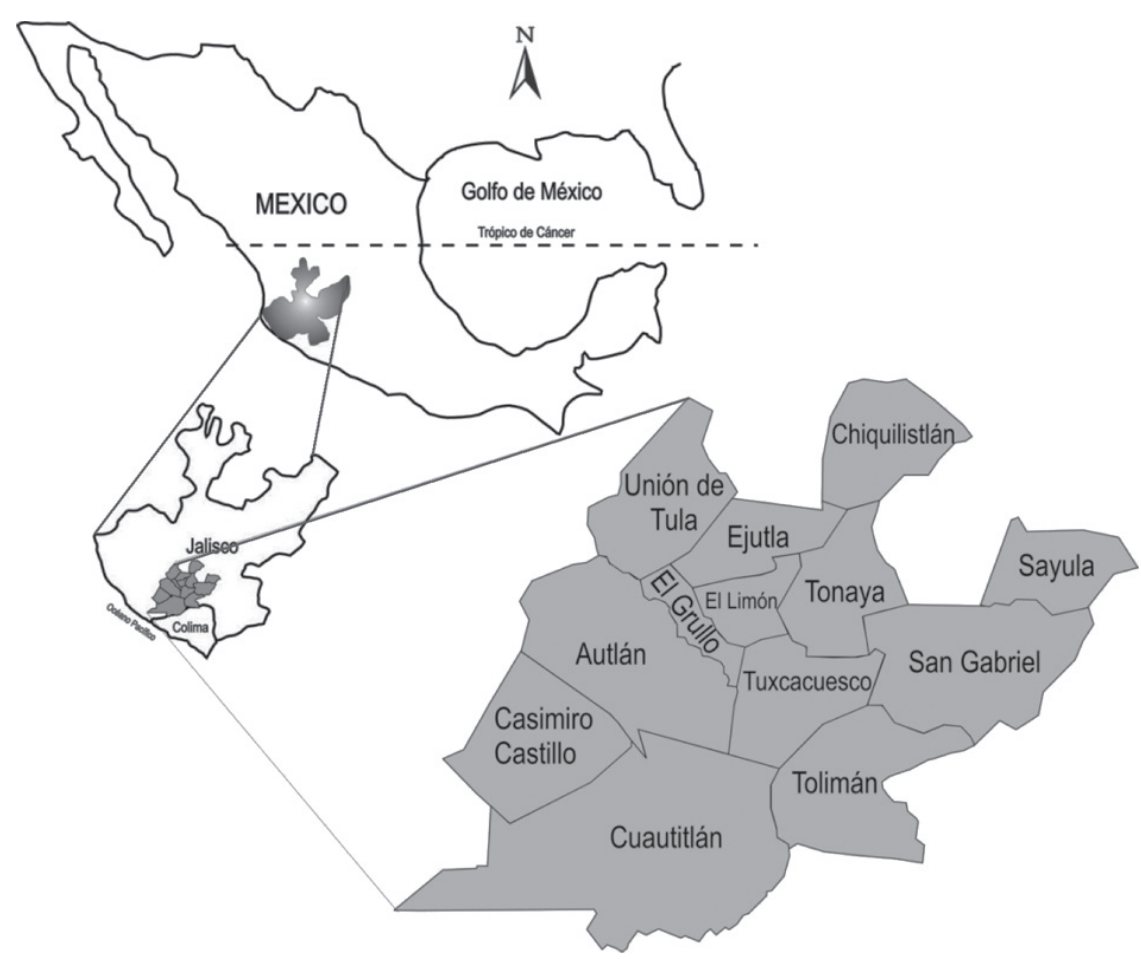

Fuente: elaborado por Gerardo Almaraz y Raquel Álvarez, Sistema Integrado de Información Geográfica, siı, Departamento de Ecología y Recursos Naturales, IMECBIO.

Globalización, sistemas de producción y sustentabilidad rural

Teóricamente, el interés en entender el problema del cultivo de agave azul tiene que ver con la relación entre las transformaciones locales, como consecuencia de los procesos de globalización y sustentabilidad rural, con un enfoque particular en los sistemas de producción. El primer proceso se refiere a la interconexión creciente 
de sistemas sociopolíticos nacionales a escala planetaria. También a los cambios sociales, económicos y ecológicos, que ocurren en los espacios locales (Waters 1995; Morales 2004), tanto en términos cuantitativos (incremento de superficie, grado de adopción entre tenencia ejidal o privada), como cualitativos (procesos sociales de transformación).

En cuanto a los cambios cualitativos, una de las repercusiones de la globalización es la reconfiguración de las relaciones sociales y políticas entre los actores, en una comunidad rural determinada (Ploeg 1994). Hoy día, en muchas de ellas se observa pobreza y deterioro de recursos naturales, y se han debilitado las bases socioproductivas. Esto indica que la globalización y su conceptualización en términos neoliberales se tiene que considerar como un proceso excluyente para una gran parte de los pobladores de una región dada (Beck 1992). Esta exclusión social ha causado problemas, como la falta de articulación de las economías campesinas (Morales 2004).

Con los efectos negativos de la globalización, el desarrollo sustentable sigue teniendo su vigencia. Por lo común, con este término se refiere al progreso que satisface las necesidades de las generaciones presentes sin comprometer las de las futuras. Sin embargo, existen muchas discusiones científicas y políticas sobre cómo hacer operativa la sustentabilidad. Los liberales, por ejemplo, mencionan que existe la posibilidad de hacer compatible el crecimiento económico con la preservación ambiental, mediante el aumento en la productividad; producir más con menos recursos y disminuir los residuos. A su vez, los ecosocialistas señalan que el capitalismo es insostenible, debido a su principio de crecimiento económico y acumulación constante. Aún con enfoques diferentes, todas las corrientes retoman al desarrollo sostenible como un concepto prometedor para revertir los problemas socioambientales (O'Connor 2000). En este artículo se toma el más conocido y aceptado en el mundo, y que hace referencia a las generaciones futuras, menciona un modelo de desarrollo visible en el aspecto económico, justo en lo social y ecológicamente apropiado.

Un sistema de producción se puede considerar como una forma específica de "hacer agricultura", es decir, se refiere a las actividades agropecuarias y forestales de los campesinos. Dichos sistemas se ba- 
san en una movilización de recursos y son el resultado del quehacer en un espacio particular, la "localidad". Es posible distinguir varios ámbitos para movilizar recursos, por ejemplo la propia parcela, la familia, la comunidad o a través de las instituciones o mercados. Por lo tanto, los sistemas de producción pueden entenderse también como configuraciones específicas de actividades productivas, económicas y sociales, y son dinámicos y variables, debido a cambios ecológicos, socioeconómicos e institucionales (Ploeg 1990).

Los actores locales pueden entender las transformaciones en los sistemas de producción como la redefinición de sus relaciones con el contexto socioeconómico e institucional, así como con el natural o material, debido a procesos sociopolíticos exógenos a la localidad (Ibid. 1992). Muchas veces estas modificaciones implican la transición del modo de producción campesino, basado en la diversificación de tareas productivas y el uso principal de recursos locales, al modo agroindustrial, basado en la especialización y el uso de recursos externos (Toledo 1995; 2000).

Una cuestión importante en el entendimiento de los sistemas campesinos de producción es su grado de sustentabilidad, lo cual se puede aproximar con indicadores cuantitativos y cualitativos. Según Ploeg (1990 y 1992); Toledo (2000) y Morales (2004), sus transformaciones por lo general conducen a una pérdida de la sustentabilidad. La incorporación de conocimientos nuevos e insumos externos puede conducir a una pérdida del conocimiento campesino, así como a la toma de decisiones en torno a las prácticas agropecuarias. Asimismo, como es el caso específico del agave azul, las alteraciones pueden llevar a un deterioro de la base material, es decir, los recursos naturales (Gerritsen y Martínez 2010). Esto se puede visualizar de forma analítica en la figura 2, donde la sustentabilidad está representada por dos variables que conjuntan aspectos ecológicos, económicos y sociales: a) estrategia productiva ("tendencia a especialización versus tendencia a diversificación”) y b) grado de endogeneidad ("uso predominante de recursos locales versus uso predominante de recursos externos”). En relación con las dos variables usadas, la figura 2 muestra cuatro posiciones básicas donde se ubican los sistemas de producción: a) el campesino, basado en la diversificación productiva, y el uso predominante de recursos 


\section{Figura 2}

Patrones normativos de desarrollo agropecuario

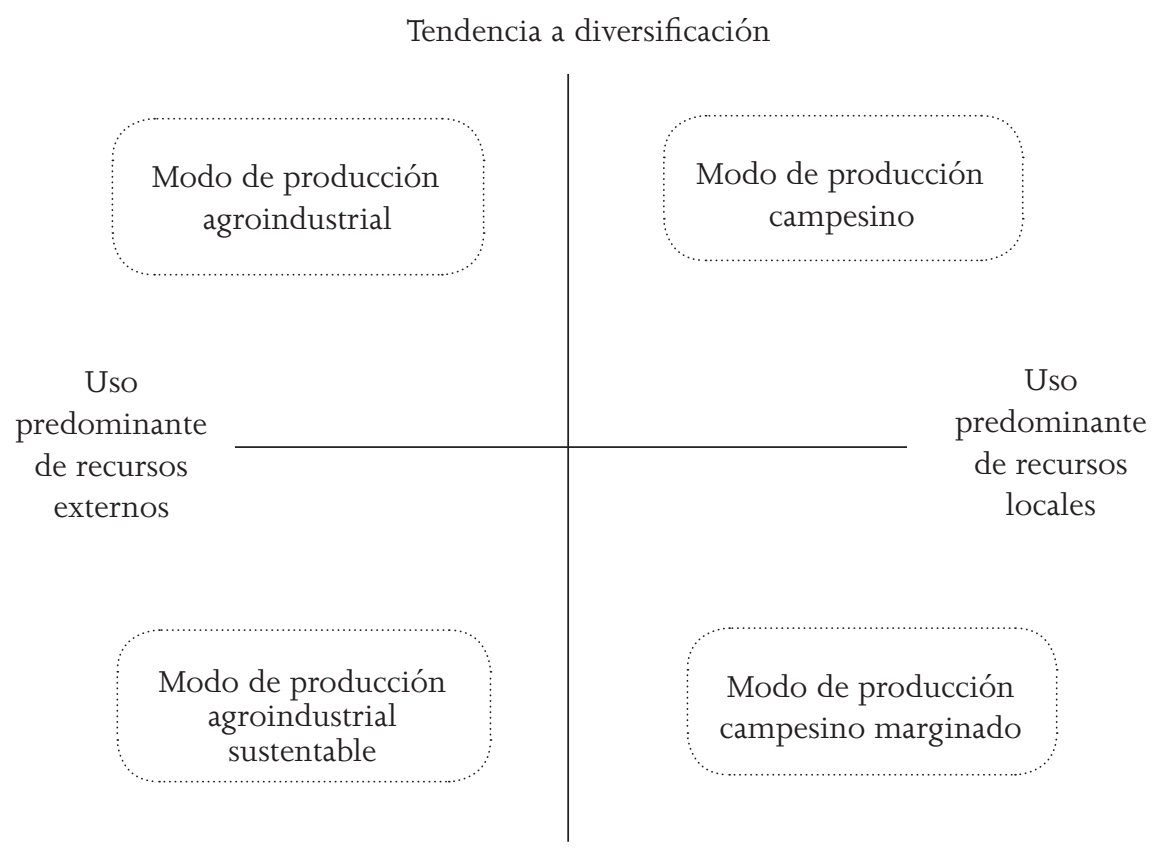

Tendencia a especialización

Fuente: adaptado de Toledo (2000), Ploeg (1990) y Gerritsen (2002).

locales; por lo tanto, contribuye a un enriquecimiento del entorno natural; b) el campesino marginado, que representa a los productores que no logran mantener una estrategia de diversificación y por lo tanto contribuyen a un empobrecimiento, o hasta degradación de su base material; c) el agroindustrial, basado en la especialización productiva, con predominio de los recursos externos, y donde se observa una tendencia de empobrecimiento de la naturaleza y d) el agroindustrial sustentable, basado en la especialización y los recursos externos, sin embargo, se busca enriquecer los recursos naturales (Gerritsen 2002). 


\section{Diseño del estudio}

Aquí se muestran datos que permiten construir un primer entendimiento de los cambios ocurridos en los sistemas productivos tradicionales de la cuenca baja del río Ayuquila, a partir de la llegada del agave azul a la zona. Además, la descripción de la evolución de este cultivo, que se presenta a continuación, es resultado de estudios realizados por los autores de este artículo y también los colaboradores Martínez et al. (2007); Bowen (2004); Nava y Medina (2005); González (2006); Durán (2006); Bowen y Gerritsen (2007); Gerritsen y Morales (2007) y Gerritsen y Martínez (2010), relacionados con este cultivo y el desarrollo rural regional. En este sentido, el presente trabajo representa el primer intento de síntesis de toda la información obtenida.

A grandes rasgos, la metodología seguida en los estudios es la siguiente:

- Revisión de la información secundaria, para obtener un panorama general del avance agropecuario en el área de estudio, incluso de la diversidad en sistemas productivos.

- Discusiones y entrevistas con informantes clave, para la comprensión cualitativa de los sistemas productivos y su contexto institucional y económico.

- Recolección de datos cualitativos y cuantitativos a través de cuestionarios estructurados, para contextualizar mejor los resultados obtenidos.

- Observación participativa, que permite contextualizar mejor los resultados obtenidos (Russell 1988).

\section{Área de estudio}

Los estudios se realizaron en los municipios de Autlán de Navarro, El Grullo, El Limón, Tonaya y Tuxcacuesco, ubicados en las regiones político-administrativas de Sierra de Amula y Costa Sur de Jalisco, en el occidente de México. Esta área se caracteriza por presentar una gran complejidad de condiciones ambientales, debido a la variación altitudinal y el relieve accidentado del terreno, y provee recursos naturales importantes para los pobladores - agua, madera, 
alimentos, forrajes, medicinas y otros no maderables, así como una variedad de servicios ambientales- (IMECBIO 2000).

Las condiciones urbanas en la región se caracterizan por un crecimiento no planificado de la zona de asentamiento. Además, existen problemas con el abastecimiento del agua potable y la descarga de las aguas residuales sin tratar en ríos y arroyos. Los índices de emigración son altos, en especial hacia Estados Unidos, lo cual induce a un proceso de aculturación sobre todo en la población joven. Las condiciones rurales muestran una riqueza cultural (por la presencia de comunidades campesinas con usos tradicionales de los recursos), pero también por un aislamiento y dispersión de los asentamientos humanos, condiciones de pobreza y marginación alta. Aun cuando las siembras básicas como las del maíz son importantes, los precios han sido bajos en los últimos años. Otros productos relevantes son la caña de azúcar, chile y jitomate, requeridos por el mercado nacional e internacional. La ganadería es significativa desde la década de 1970 y el agave azul desde la de los años noventa (Gerritsen et al. 2005).

\section{Expansión del cultivo de agave azul}

La importancia del cultivo de agave azul ha aumentado en la cuenca del río Ayuquila, desde 1994 (Gerritsen y Martínez 2010). Por tradición, en Tonaya y los municipios cercanos de Tolimán, Tuxcacuesco y Zapotitlán de Vadillo se sembraba el agave verde (Agave angustifolia Haw), utilizado desde hace siglos en la elaboración de una bebida tradicional conocida antes como "vino", "mezcal" y ahora como "licor de agave" (Colunga y Zizumbo 2006). Sin embargo, la demanda para el agave azul, empleado en las agroindustrias tequileras del centro y de los Altos de Jalisco ha ocasionado un incremento dramático en Amula y la Costa Sur y la transformación de los sistemas de producción tradicionales de otros cultivos, con el desplazamiento consecuente de las variedades locales y de las prácticas agrícolas culturales.

La figura 3 muestra la evolución del agave azul en los municipios de estudio, y la 4 las superficies sembradas por año. Cabe mencionar 
que no está considerada el área cosechada; si bien en 2004 apenas terminó el segundo ciclo del agave en la región, es probable que la superficie real sembrada sea un poco menor a la representada en la figura 3. Asimismo, en el caso de El Limón y Tuxcacuesco sólo existen datos para el lapso 1996-2002, y para El Grullo de 1998 a 2002. Por otro lado, para Tuxcacuesco falta información; el total del agave plantado fue de 359.4 hectáreas, pero hubo 490.9 registradas.

\section{Figura 3}

Superficie acumulada del agave plantado por municipio

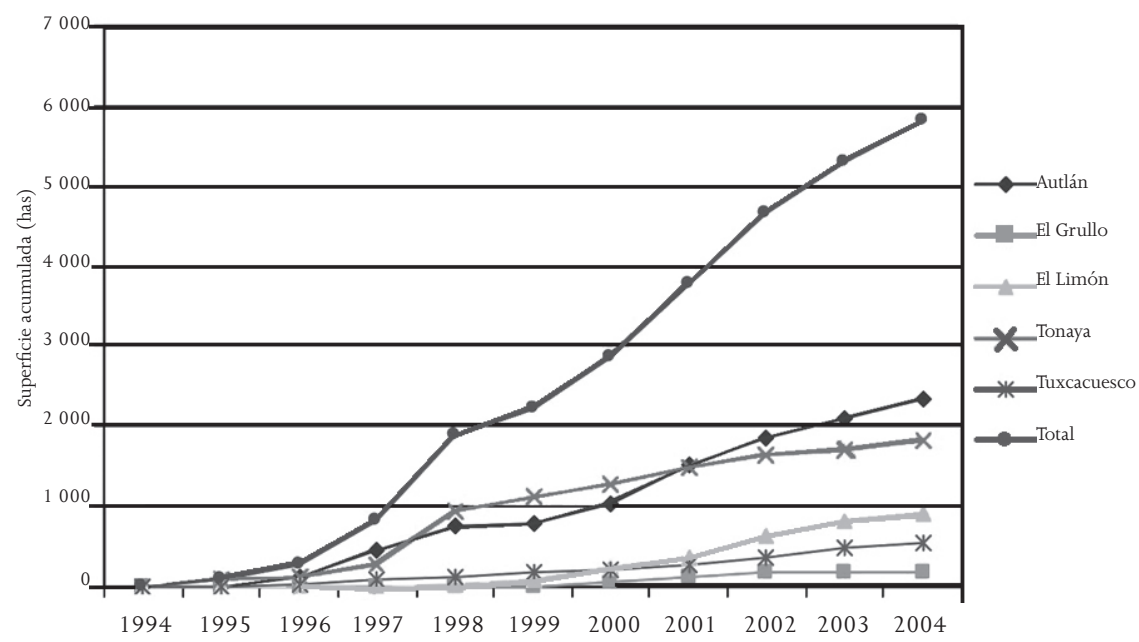

Fuente: elaboración propia, con base en datos del proyecto "Competitividad agropecuaria y desarrollo rural sostenible”, CADERS, Autlán de Navarro y El Limón.

De 1998 a 2001, el auge del agave azul ocurrió en especial en Autlán de Navarro y Tonaya. La figura 4 demuestra que en diez años la superficie sembrada creció de manera exponencial, aunque con una disminución drástica en el área que se plantó en 1999 en relación con los años anteriores y posteriores (véase figura 5). Según Zamora y Preciado (2003), esta disminución se debió a la falta de hijuelos para el establecimiento de plantaciones nuevas, debido a 
la incidencia elevada de plagas en las zonas tradicionales en Amatitán, Arandas y Tequila, así como al rechazo de los productores de las regiones Centro y los Altos ante los contratos ofrecidos por las industrias tequileras (Valenzuela y Gerritsen 2007).

\section{Figura 4}

Superficie del agave plantado por año por municipio

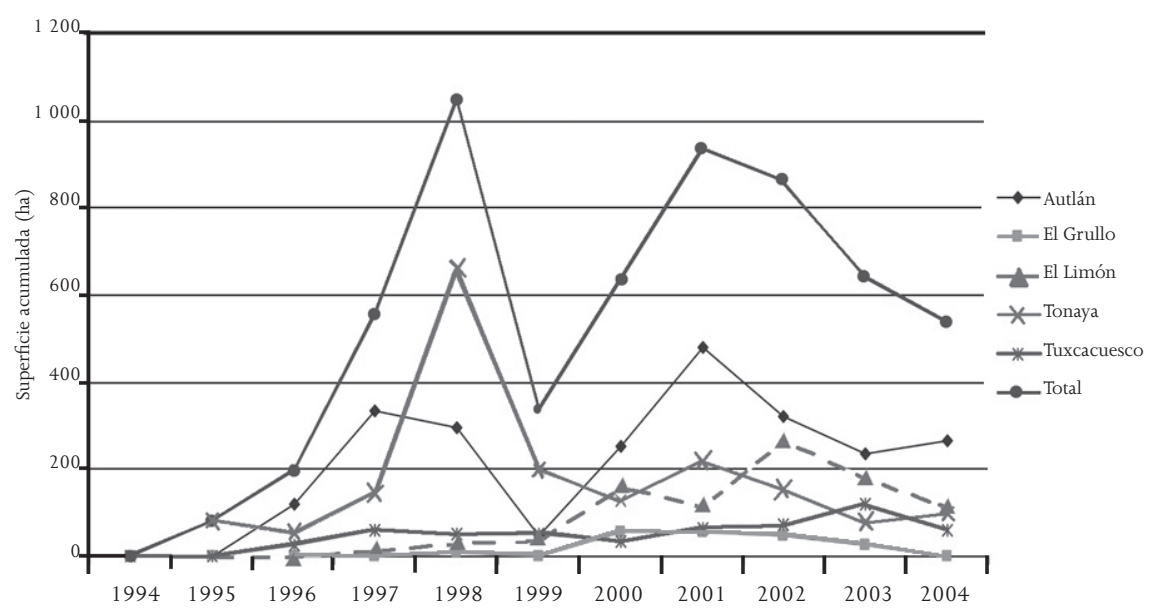

Fuente: elaboración propia, con en base en datos del CADERS, Autlán de Navarro y El Limón.

La figura 4 muestra una imagen de la dinámica de expansión del agave azul, y permite notar que Tonaya tenía más agave en 1997, mientras que en Autlán fue mayor al principio (1997-1998) y al final de la primera década del cultivo en la región (1994-2004). Un caso interesante es El Limón que mostró un crecimiento significativo en la segunda mitad de la década estudiada, es decir, 1999-2000. Después de disminuir la plantación en 1999 en todos los municipios, el área con agave siguió aumentando, aunque con menos superficie plantada. Por último, la figura 4 indica una disminución de agave sembrado a partir de 2002, quizá ocasionado por la crisis actual de precios para este cultivo. 
En el periodo 1994-2004, como lo indica la figura 5, la superficie sembrada con agave azul comprende entre 1.9 y 10.8 por ciento del total de las unidades rurales de producción. Si bien Autlán de Navarro y Tonaya han tenido el crecimiento más acelerado, en El Limón es donde se observa el mayor cambio de uso de suelo, con casi 11 por ciento.

\section{Figura 5}

Superficie total sembrada de agave en 1994-2004

\begin{tabular}{|l|c|c|c|}
\hline \multicolumn{1}{|c|}{ Municipio } & $\begin{array}{c}\text { Superficie unidad de } \\
\text { producción rural(ha) }\end{array}$ & $\begin{array}{c}\text { Superficie agave } \\
\text { (ha) }\end{array}$ & Porcentaje \\
\hline Autlán de Navarro & 30174.8 & 2350.74 & 7.8 \\
\hline El Grullo & 10224.0 & 195.7 & 1.9 \\
\hline El Limón & 8518.1 & 923.35 & 10.8 \\
\hline Tonaya & 21632.1 & 1814.05 & 8.4 \\
\hline Tuxcacuesco & 19717.7 & 536.11 & 2.7 \\
\hline Total & 90266.7 & 5819.95 & 6.4 \\
\hline
\end{tabular}

Fuente: elaboración propia, con base en Gerritsen et al. (2005) y datos del CADERS, Autlán de Navarro y El Limón.

\section{Agave azul y cambio de uso de suelo}

El cultivo de agave verde en esta región de Jalisco ha sido una opción en cuanto a la evolución de los sistemas de producción desde hace más de un siglo, sin embargo en la última década, el agave azul ha inundado las áreas agrícolas de la zona. Este cultivo nuevo ha originado un desplazamiento de los granos básicos (maíz y sorgo), incluso cambios en la ganadería. Como consecuencia se observa una transformación parcial de los sistemas tradicionales de producción en esta parte del estado; los factores responsables se presentan más adelante. La figura 6 ejemplifica la sustitución de cultivos tradicionales por el agave azul en Tonaya (Martínez et al. 2007), tendencia que Hostettler (2007) confirma para Autlán. 
Figura 6

Tendencias de cambio de uso del suelo en Tonaya

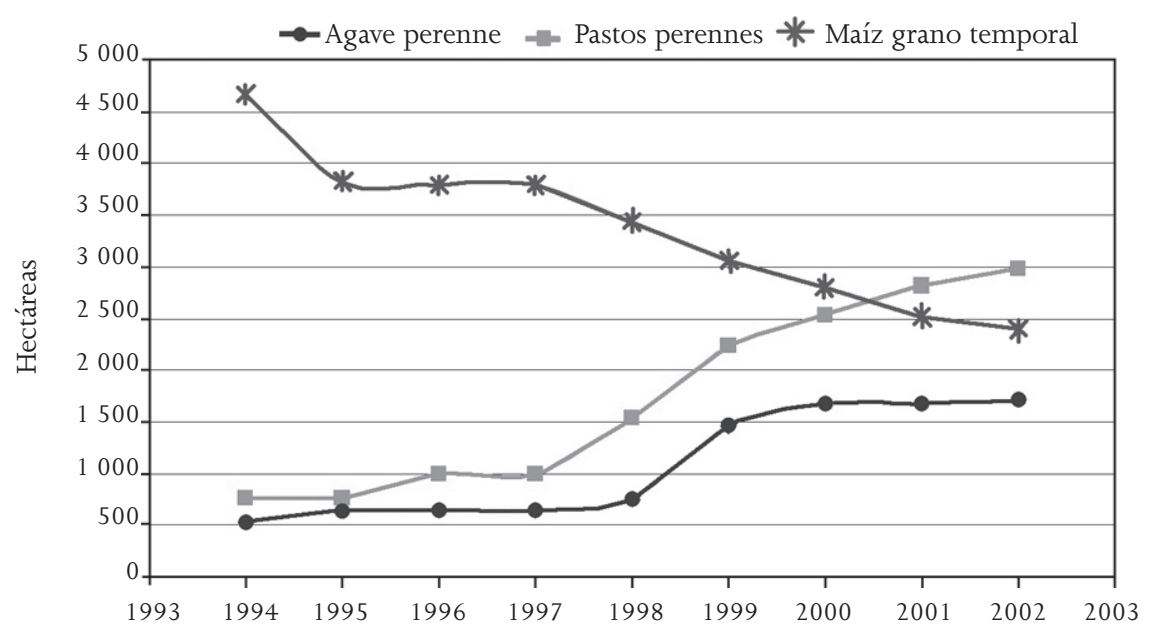

Fuente: Martínez et al. (2007).

\section{Agave azul y tenencia de la tierra}

En tanto al régimen de propiedad, los porcentajes relativos de la superficie con tenencia privada y ejidal del agave azul varían entre los cinco municipios, aunque se distinguen dos grupos grandes (véase figura 7). Por un lado, en Autlán de Navarro, El Grullo y El Limón predomina la siembra entre los ejidatarios, mientras que en Tonaya y Tuxcacuesco hay una cantidad considerable de propietarios privados. No obstante, la figura 8 muestra que, excepto por los productores privados de Tonaya, la mayoría tiene superficies relativamente pequeñas plantadas con agave azul. Cabe mencionar que para el caso de Tonaya, los productores cultivan agave azul y verde. 


\section{Figura 7}

Caracterización de productores de agave azul según tenencia de la tierra

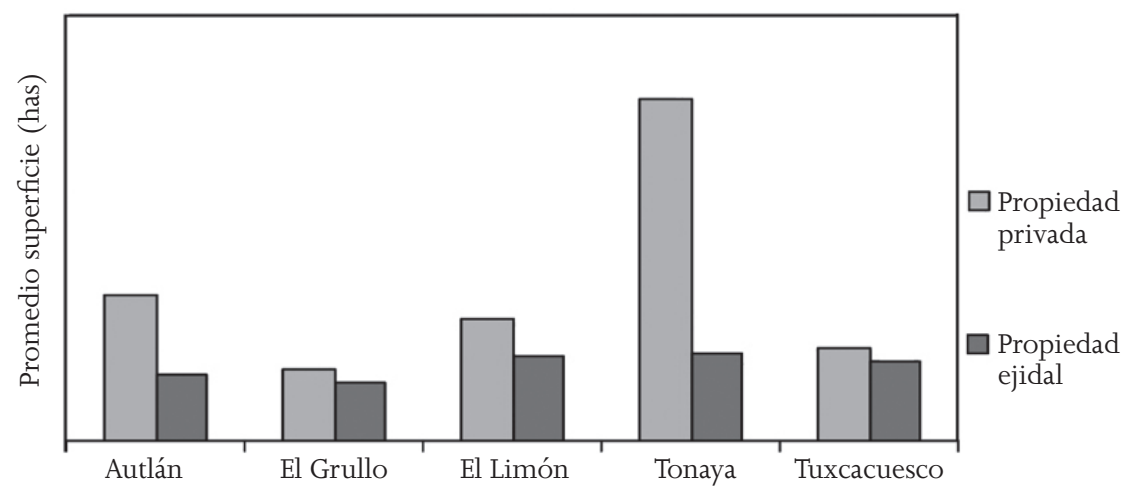

Figura 8

Promedio de la superficie con agave azul, según tenencia de la tierra

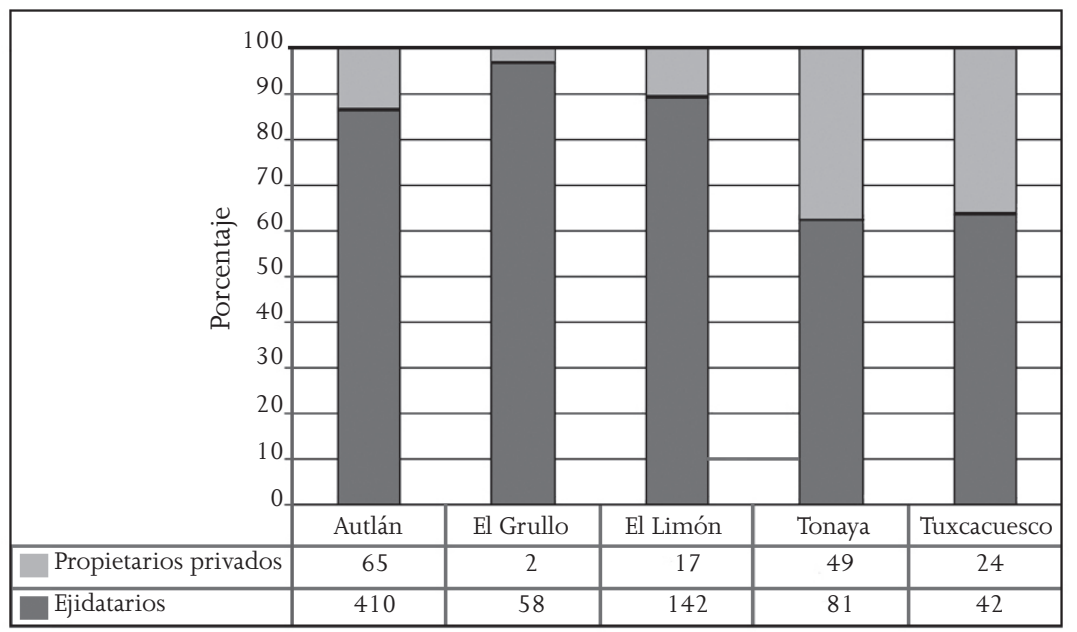

Agave azul y el sistema de cultivo

En la cuenca baja del río Ayuquila es característico el sistema de monocultivo, centrado en el agave azul, por ser perenne (con un 
ciclo de siete a ocho años), como en la zona de origen y en la región alteña, la densidad de siembra supera las 3 mil plantas por hectárea, y para la preparación de la tierra se practican labores mecánicas, sin considerar la pendiente del terreno. Asimismo, destaca el uso de controladores químicos para "malezas" y plagas de insectos. Los agroquímicos utilizados para contrarrestar las plagas y enfermedades son Carborufan, sulfato de cobre, Lambda cyhalotrina, Metomilo, Clorpirifos, Monocrotofos, Mancozeb, Furadan y diazinon (Zamora y Preciado 2003; Nava y Medina 2005).

Los fertilizantes principales empleados son urea, sulfato de amonio y nitrato de amonio, aunque también se usan otras fórmulas derivadas de análisis de suelos. Los herbicidas más comunes son Diurón, diglifosfato, ametrina, bromacil, oxamil, tebuthiuron, picloram, ácido 2-4D y Paraquat (Ibid.). La incidencia de plagas y enfermedades en el agave azul se acentúa con los ciclos de sobreproducción (García 1997), en casi todos los casos el uso de agroquímicos es una práctica obligada aunque en ocasiones existen técnicas de prevención, que permiten reducir la incidencia de enfermedades (Ibid.; Valenzuela 2003).

En el manejo de la plantación es necesaria la poda para lograr varios objetivos, entre los que destacan el barbeo, poda de semilla (hijuelos), cacheteo o chaponeo, podas fitosanitarias de formación y maduración (Ibid.). El desquiote, capado o corte del escapo floral se realiza en plantas maduras, que comienzan a florecer alrededor del quinto o sexto y hasta el octavo año, para mantener la producción de azúcares en la piña. Por lo regular, el quiote se corta cuando alcanza los 50 centímetros, "la corta" se realiza de forma manual con la coa de jima, barretón, machete o hacha. En caso de que los agaves no sean capados, la inflorescencia consume todos los polisacáridos que contiene la cabeza para la producción sexual de plantas nuevas, actividad realizada muy rara vez (Ibid.).

La jima (cosecha) depende de la madurez de la planta, y ésta a la vez en gran medida de las condiciones del suelo, el clima y las atenciones culturales al cultivo (García 1997). Algunos indicadores de la madurez son el amarillamiento, la aparición del escapo floral o quiote y en ocasiones de mieles en las hojas. 


\section{Agave azul y sistemas de producción}

Como lo señala González (2002), si bien existen agricultores especializados sólo en el cultivo de agave azul, la mayoría lo ha incorporado en su sistema de producción tradicional, donde predomina cierta diversificación (Bowen 2004). La figura 9 ejemplifica algunos grados de diversidad productiva relacionados con el cultivo de agave azul en Tonaya, que se pueden encontrar también en otros municipios (Gerritsen y Martínez 2010).

\section{Figura 9}

Representación esquemática y ubicación de los sistemas de producción que incluyen agave azul en Tonaya

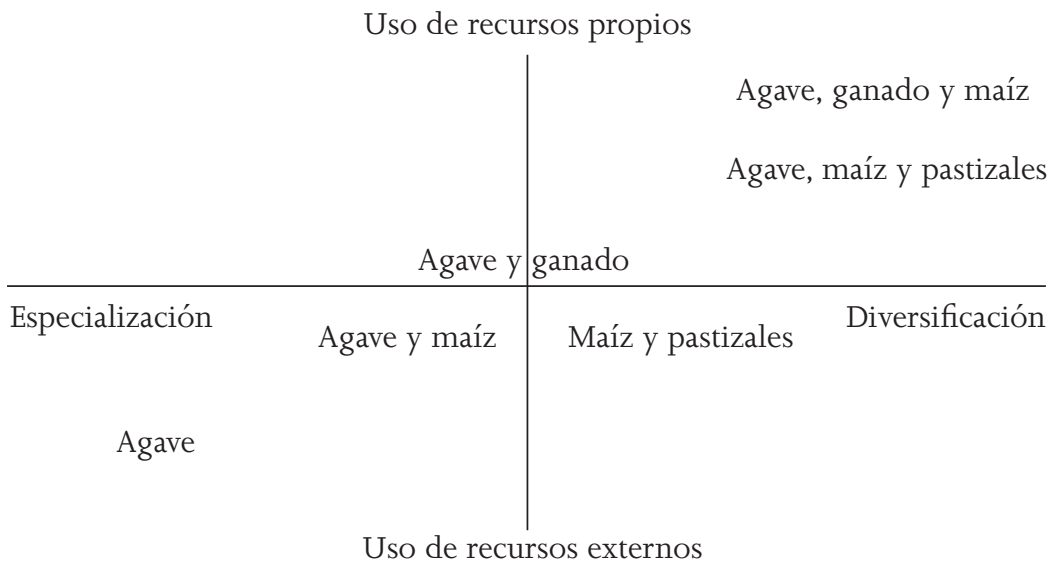

Fuente: Durán $(2006,67)$.

Agave azul y agricultura de contrato

En los sistemas de producción privada o ejidal hay agricultores que se vincularon con empresas tequileras, mediante contratos de arren- 
damiento. Las razones para hacerlo fueron, por un lado, el beneficio y la seguridad económica que brindan, sobre todo para quienes no cuentan con capital suficiente para invertir en el establecimiento del agave azul; por otro, como el cultivo es nuevo en la región y como la agricultura de contrato se basa en la trasferencia de paquetes tecnológicos, en ellos los productores encontraron un beneficio económico (véase figura 10) (Martínez et al.2007; Bowen y Gerritsen 2007).

Figura 10

Algunas ventajas y desventajas de la agricultura de contrato

\begin{tabular}{|l|l|}
\hline Agricultura con contrato de renta & Agricultura independiente \\
\hline Mercado asegurado & Mercado inseguro \\
Paquetes tecnológicos & Producción más tradicional \\
Seguridad económica, pero falta de respaldo legal & Más desconocimiento \\
\hline
\end{tabular}

La naturaleza de los contratos ha cambiado a través de los años; en el ciclo 1995-1996, la empresa agavera ofrecía mejores condiciones, además de pagar la renta anual equivalente al valor de una tonelada de maíz por hectárea a precios de enero de cada año, también otorgaba al productor una participación de la cosecha total de un máximo de 5 por ciento al momento de la jima (cosecha). Destaca que al principio los campesinos rentaban las tierras marginales (coamiles y parcelas degradadas). En la actualidad se ofrece la renta correspondiente al valor de dos toneladas de maíz al año, con un precio mínimo de 1300 pesos por tonelada, y sólo para terrenos de muy buena calidad (planos y fértiles) se otorgan porcentajes máximos de 3 por ciento al momento de la jima (Gerritsen y Martínez 2010).

Nunca ha existido autoridad alguna que dé fe de los contratos (ejidales, municipales, otros), mucho menos son notariados, para que "obliguen" de alguna forma a cumplirlos por ley; por ende, se trata de simples acuerdos escritos con testigos de "ambas" partes. En dicho contrato sobresale una de las cláusulas: "los beneficios 
que queden perdurables, quedarán a favor del arrendador al término del presente contrato". Sin embargo, en ninguna se menciona quién paga los daños ocasionados durante el cultivo (erosión, contaminación, otros). Según esta lógica, el arrendador (dueño de la tierra) también se queda con los "daños perdurables" (Martínez et al. 2007).

Factores que influyen en la expansión de agave azul

Son varios los factores que influyen en la expansión del agave azul en la cuenca baja del río Ayuquila; por lo general constituyen un conjunto que rige el cultivo del agave, ya sea por contrato o de manera independiente. Bowen (2004) y Bowen y Gerritsen (2007), basándose en el municipio de Autlán de Navarro mencionan los siguientes:

- Factores biofísicos

El agave, a diferencia del maíz, requiere poca lluvia. La variabilidad en frecuencia y cantidad en la precipitación, observada en la Costa Sur de Jalisco, es una de las razones expuestas por los productores para decidirse a sembrar agave azul, y también la ausencia de sistemas tecnificados de irrigación.

\section{- Factores económicos}

La economía es uno de los elementos más importantes para predecir cuáles agricultores arrendarán sus tierras y quiénes podrán cultivar agave por sí mismos. Casi todos coinciden en que elegirán utilizar sus tierras en lugar de rentarlas. La mayoría de los que rentaron señala a la restricción financiera como la razón principal para hacerlo. Además, sembrar agave azul requiere grandes inversiones, desde su plantación hasta la cosecha; costos que los campesinos no pueden solventar. Rentar una parcela es mucho menos redituable que las ganancias que podrían obtener del cultivo independiente. Sin embargo, aunque el pago por la renta es muy bajo, es aceptable 
y atractivo, porque no hay inversión monetaria ni de trabajo, y en cambio se recibe una cantidad anual estable. A ello se suman los ingresos por subsidios agrícolas como el del Programa de Apoyos Directos al Campo (PROCAMPO). Este factor influye en la expansión del cultivo del agave de dos maneras: motiva a los productores con pocos recursos financieros a rentar sus tierras, y les permite a quienes tienen más capital comenzar a sembrar agave en forma independiente. Estos últimos financian su cultivo con ahorros, salarios o con las ganancias de otras cosechas. También los envíos de dinero de los emigrantes empleados en Estados Unidos juegan un papel importante, y les permiten a algunos trabajar por sí mismos.

- Disponibilidad de trabajo

Un factor esencial para arrendar las tierras a las empresas tequileras es la disponibilidad de trabajo; en especial para los agricultores de edad avanzada, quienes son físicamente menos capaces, y los contratos les ofrecen un ingreso anual, aun cuando los términos sean más desfavorables.

- Riesgos

Otro de los elementos principales es minimizar o trasferir los riesgos financieros y ambientales. Como el precio del agave ha sido muy variable, los contratos representan un tipo de seguro contra esta variabilidad financiera (González 2002). Por otro lado, al rentar la parcela, de cierto modo los productores evitan los riesgos ambientales, como las condiciones climáticas adversas y las infecciones virales, ya que los "trasfieren" al contratista. La percepción sobre los riesgos difiere de acuerdo a los recursos financieros de cada productor; los que tienen grandes extensiones de terreno e insumos adicionales, lo dejan a la suerte; mientras que quienes cuentan con poco capital se preocupan más, debido a la variabilidad en los precios, y de si venderán o no sus cosechas. Los riesgos son un factor importante para los productores con menos acceso a recursos financieros, y juegan un rol positivo en la toma de decisiones para rentar sus tierras. 
- Factores institucionales

En 1997 se incluyó el cultivo de agave en PROCAMPO, hecho que aumentó la posibilidad de que los productores comenzaran a sembrar más. Por otra parte, la entrada de las industrias tequileras les facilitó el arrendamiento de tierras a intermediarios. La decisión de adoptar un cultivo nuevo está muy vinculada con la existencia de instituciones que mitigarán los riesgos asociados. Con la entrada del Tratado de Libre Comercio de América del Norte, entre 1994 y 2002, los subsidios agrícolas disminuyeron por más de un tercio, y hubo precios bajos para algunos cultivos. La combinación de la renta y la ayuda de PROCAMPo les dio un ingreso, aun cuando fue mínimo.

Las compañías tequileras pueden controlar los precios de la venta del agave. Para combatir estas restricciones institucionales, los productores formaron la Asociación Agrícola de Agaveros en Autlán de Navarro, que trabaja para asegurar un precio alto y encontrar compradores con facilidad. Existe también el Consejo Consultivo Regional para la Cadena Productiva del Agave en la Sierra de Amula (que incluye a Autlán de Navarro), que estabiliza el precio y minimiza los ciclos de oferta y demanda. Las asociaciones proveen soporte técnico e información sobre la producción de agave. Antes de la entrada de las tequileras, a mediados de los años noventa, la mayoría de los campesinos en el área nunca habían considerado el agave como un cultivo potencial, desde entonces la única información provenía de los contratistas independientes; la introducción de recursos nuevos de información es muy importante tanto para el bienestar financiero de los particulares en la región, como desde una perspectiva ambiental. Los agricultores parecen optimistas ante el potencial de estas asociaciones para ayudar a aliviar el desequilibrio del poder y la pérdida de comunicación entre las industrias tequileras y los productores independientes de agave.

\section{- Percepciones ambientales}

Por último, otro factor que puede jugar un papel en la expansión del cultivo de agave azul es el modo en el que los campesinos perciben los efectos ecológicos del cambio de uso del suelo. Estudios han 
demostrado consecuencias negativas considerables, ya que por ser un monocultivo es vulnerable a enfermedades por lo que requiere el uso masivo de pesticidas e insecticidas; visto como una pérdida de biodiversidad (Martínez et al. 2007). También incrementa la erosión, ya que por lo común se siembra en suelo desnudo y a favor de la pendiente, lo cual ocasiona pérdida de suelo al dejarlo sin su capa arable y completamente degradado (Gutiérrez 2005). No obstante los riesgos, los productores eligen cultivar agave azul, por lo que es importante examinar su concepción sobre los daños posibles.

De acuerdo con un estudio de las percepciones de los agricultores en el ejido de Mezquitán, municipio de Autlán de Navarro, 25 por ciento indicó que la posibilidad de daños ambientales serios es rara o poco probable, mencionaron que no hay evidencia de algún efecto negativo en sus tierras; 50 por ciento reconoció que el agave azul podría tener efectos ambientales negativos, pero que no les perjudican; algunos con poco dinero señalaron que se sintieron obligados por su situación financiera, aun si el agave azul causara daño, pues tienen pocas opciones: rentar sus tierras o seguir sembrándolo (Bowen 2004). Los efectos ambientales más notorios son de los químicos usados por los contratistas, pues han destruido los árboles aledaños a las áreas de cultivo. El resto cree que la expansión del cultivo tendría consecuencias ambientales graves. Muchos dijeron que los contratistas no se responsabilizaban por la tierra, por lo que adoptan métodos nocivos. La confusión y falta de información sobre las consecuencias ambientales posibles de sembrar agave azul parece estar fomentando cada vez más la renta de tierra a los contratistas independientes (Ibid.).

\section{Agave azul, cadena productiva y denominación de origen del tequila}

Si bien el agave azul puede ser un cultivo comercial importante para el desarrollo regional, su producción carece de una buena planificación y las irregularidades en la oferta provocan fluctuaciones severas de precios (Leclert et al. 2010). En 2004, las plantaciones de 
agave se redujeron por efecto de las bajas expectativas en los precios del mismo (véase figura 4), en este sentido los productores actúan en relación directa sin tomar en cuenta que el agave es de ciclo largo (seis a ocho años), por lo que los efectos de precios se reflejan en forma inversa; esto es, si existe poca superficie sembrada en 2004 y 2005 y con el supuesto que continuará incrementándose la demanda de las bebidas, para 2011 y 2012 existirá de nuevo escasez, y por lo tanto habrá un aumento en los precios. Este problema se puede evitar si las autoridades, en conjunto con los productores de agave azul y las industrias, planifican la producción con la finalidad de que todos se beneficien.

Una de las respuestas locales a esta crisis de precios es el aumento en el establecimiento de destilerías y la producción de licor de agave en la cuenca baja del río Ayuquila (véase figuras 11 y 12) (Leclert 2007). Como también se mencionó, en la región existe una tradición de producción de licor de agave de por lo menos un siglo.

Figura 11

Evolución del número de destilerías en la cuenca baja del río Ayuquila

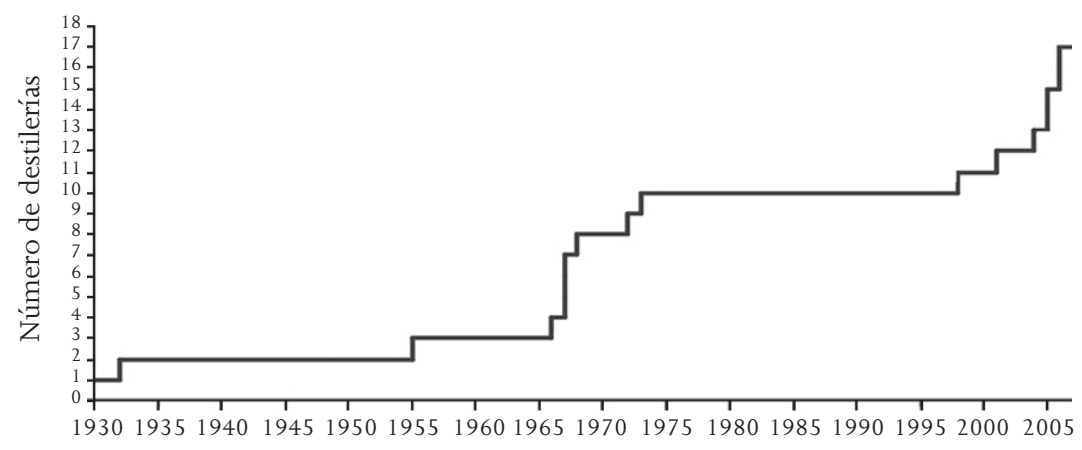

Fuente: Gerritsen et al. (2007).

Si bien se observa un crecimiento sustancial de las destilerías locales, la sobreproducción de agave azul es de tal magnitud que 


\section{Figura 12}

Elaboración aproximada de licor de agave (38/55\%)

en la cuenca baja del río Ayuquila

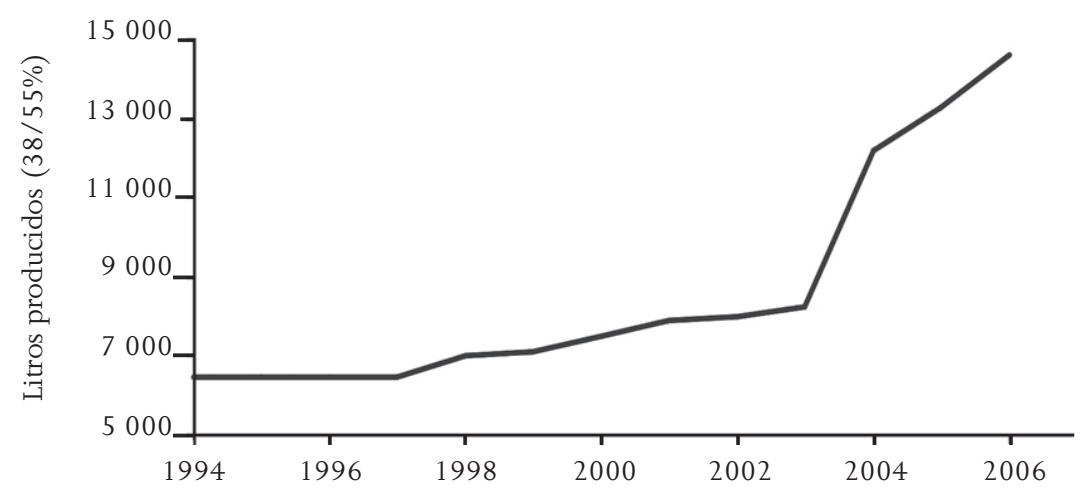

Fuente: Gerritsen et al. (2007).

muchos productores no tienen mercado para su producto (Leclert 2007). En este sentido, la indicación geográfica para el tequila, es decir, la denominación de origen del producto, que entre otras debería incluir un beneficio para los productores de agave azul, resulte en un efecto contrario, y las manifestaciones sociales de la denominación son muy generales o negativas (véase figura 13) (Schweighoefer 2007).

\section{Figura 13}

Manifestaciones sociales de la denominación de origen en la cuenca baja del río Ayuquila

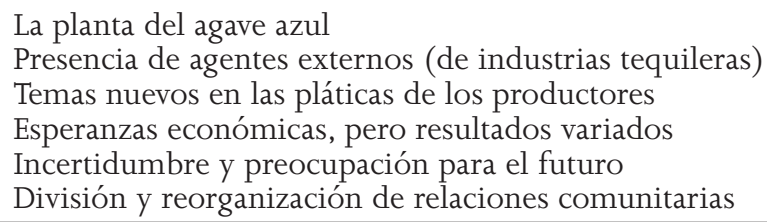

Fuente: Schweighoefer (2007). 


\section{Recomendaciones y propuestas}

de manejo sustentable del cultivo de agave

En esta sección se relaciona la expansión del agave azul con el desarrollo rural sustentable. Se ubican los sistemas de producción en el contexto de dicho desarrollo, y es importante reconocer que la sustentabilidad es un fenómeno multidimensional, que abarca aspectos ecológicos, económicos y sociales altamente interrelacionados, por tanto no es fragmentario ni está aislado (Morales 2004). Por eso se presentan algunas recomendaciones para incrementar la sustentabilidad del cultivo del agave azul y sus sistemas productivos relacionados, según los factores que por lo general se distinguen en los esquemas de desarrollo sustentable.

\section{Recomendaciones ecológicas}

- Comparación de los sistemas de producción del agave verde (nativo) y azul (introducido), para identificar las prácticas agropecuarias sustentables.

- Disminución del uso de herbicidas, a través de la contratación de mano de obra local o de la introducción de otras prácticas, que reduzcan el empleo de estos pesticidas (como los cultivos de cobertura, por ejemplo).

- Experimentación con sistemas de producción de agave que incorporen otras especies en asociación (cultivos de cobertura, asociaciones de cultivos, etcétera).

- Fomento de la producción agroecológica y orgánica del agave, así como de su destilación.

- Mantenimiento de las especies leñosas en los límites de las parcelas, que favorezcan las áreas verdes y sirvan de hábitat y corredores biológicos para la fauna silvestre.

- Establecimiento del cultivo del agave sobre surcado al contorno, para suelos con pendientes entre 2 a 5 por ciento y sobre terrazas con inclinaciones mayores de 5 . Además de coberturas de suelo entre líneas de cultivo.

- Restricción del establecimiento del cultivo del agave azul en áreas con susceptibilidad alta a la erosión, o limitar el uso en tanto no se observen medidas que mitiguen efectos negativos potenciales. 
- Conservación de las coberturas vegetales entre hileras de agave, puede ser en verde o muertas (pasto seco, residuos de cultivos).

Recomendaciones económicas

- Análisis del contexto económico nacional e internacional, para la identificación de perspectivas del mercado de tequila a mediano y largo plazo.

- Análisis de las posibilidades para la elaboración de una denominación especial (indicación geográfica, marca colectiva), para el licor de agave producido con plantas nativas en los municipios de estudio y otras áreas aledañas.

- Evaluación del efecto económico del cultivo de agave en comparación con otros, incluso un análisis de las perspectivas a mediano y largo plazo.

- Esquemas de incentivos para agricultores que conserven o promuevan la producción "limpia" u orgánica, así como la diversidad biológica del agave.

Recomendaciones sociales

- Especificar, en la elaboración de contratos, que el arrendador se comprometa a retribuirle los costos de degradación de los recursos al dueño de la parcela, si fuera el caso.

- Analizar los actores involucrados en el cultivo de agave azul y su inclusión posterior en el consejo municipal, para la cadena productiva.

- Fortalecer la toma de decisiones locales.

- Fomentar la capacidad de gestión de los productores locales.

- Promover la inclusión y organización entre agricultores, que permita la cohesión en la producción de agave azul.

Recomendaciones político-institucionales

- Redirección de subsidios gubernamentales, para el fomento de una producción sustentable de agave azul. 
- Regularización del uso de los herbicidas disponibles en el mercado, permitidos en la norma y la legislación.

- Regularización de la apertura de áreas de bosque para dedicarlas al cultivo de agave en el municipio, aplicando eficientemente las leyes y normas sobre la materia.

- Elaboración de una clasificación de tierras a escala regional, para disponerlas de acuerdo a su potencial para la producción del agave azul, con base en las condiciones de suelo (fertilidad, profundidad, susceptibilidad a la erosión, etcétera), clima y elementos productivos.

- Regulación, a través de los consejos consultivos municipales y regionales, de la planificación del cultivo del agave junto con el Consejo Regulador del Tequila.

\section{Conclusiones}

Aquí se presentó una aproximación de la dinámica socio-productiva del agave azul introducido en los años noventa en cinco municipios de la cuenca baja del río Ayuquila, sobre todo por firmas tequileras. Queda claro que la expansión de la planta en esta región es diferenciada; en algunos municipios ha sido más acelerada que en otros. Además, los resultados indican que su introducción ha sido desigual, es decir, si bien hubo una gran adopción del cultivo, la mayoría de los productores no abandonaron por completo sus actividades tradicionales.

Para retomar las nociones teóricas de la primera parte del artículo, se puede concluir que el cultivo del agave azul representa el sistema productivo agroindustrial (véase figura 13), lo cual se caracteriza por un grado alto de especialización y gran dependencia de insumos externos (Toledo 1995 y 2000; Ploeg 1992). La expansión del agave azul repercute de manera profunda en la sustentabilidad rural del área de estudio, sobre todo cuando se siembra por contrato, como lo han reportado también otros trabajos (Colunga et al. 2007; Gerritsen y Martínez 2010).

El desafío de los sistemas de producción agroindustriales, caracterizados por una sustentabilidad rural menor, o donde existe un 
riesgo de afectar a la sustentabilidad regional, está en aumentar su grado de sustentabilidad, es decir, disminuyendo su impacto socioambiental negativo. En este sentido, las recomendaciones y propuestas son un primer paso en la recuperación y fortalecimiento de la sustentabilidad regional.

\section{Figura 13}

Representación esquemática del agave azul

y su evolución agropecuaria

Tendencia a diversificación

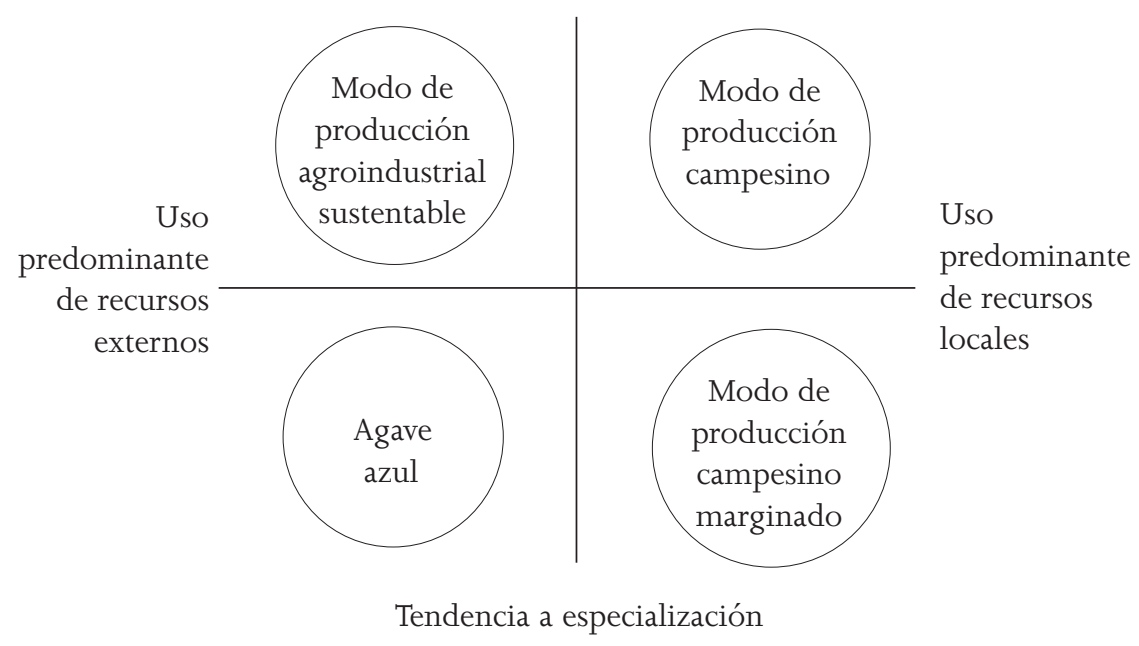

Fuente: elaboración propia, con base en la figura 2.

La puesta en marcha de esquemas de intervención, para aumentar la sustentabilidad de los sistemas de producción basados en el agave azul, requiere la participación activa de los productores y demás actores institucionales de la zona (ayuntamientos, organismos estatales y federales) (Gerritsen y Morales 2007; Gerritsen y Martínez 2010). En este sentido, los consejos consultivos municipales y 
regionales para la cadena productiva de agave azul son un importante primer paso como parte de las acciones derivadas de la nueva Ley de Desarrollo Rural Sustentable.

Sin embargo, se requiere también de otros instrumentos y medidas que permitan crear un contexto institucional favorable para el desarrollo regional sustentable, no sólo del cultivo de agave azul, sino de todos los presentes (Pretty 1995). Por último, cualquier esquema de intervención tendrá que asegurar tanto el desarrollo regional económico como la conservación de los recursos naturales (Gerritsen y Martínez 2010).

Recibido en enero de 2010 Aceptado en abril de 2010

\section{Bibliografía}

Beck, Ulrich. 1992. The Risk Society. Towards a New Modernity. Londres, Thousand Oaks, Nueva Delhi: sage Publications.

Bowen, Sarah. 2004.The Road to Margaritaville: Expansion of Agave Cultivation and Power Dynamics in Southern Jalisco, Mexico. Tesis de maestría en sociología. Department of Rural Sociology, University of Wisconsin-Madison, Wisconsin.

y Peter R.W. Gerritsen. 2007. Reverse Leasing and Power Dynamics Among Agave Farmers in Western Mexico. Agriculture and HumanValues 24: 473-488.

Ana Livier Durán Amaya y Peter R.W. Gerritsen. 2004. Análisis cuantitativo del agave en los municipios Autlán, El Grullo, El Limón, Tonaya, Tuxcacuesco. Informe interno. Autlán: Universidad de Guadalajara.

Colunga, Patricia y Daniel Zizumbo. 2006. Tequila and other Agave Spirits from West-central Mexico: Current Germplasm Diversity, Conservation and Origin. Biodiversity Conservation 1-15. 
y L. Eguiarte. 2007. En lo ancestral hay futuro: del tequila, los mezcales y otros agaves. Mérida: Centro de Investigación Científica de Yucatán (CICY), Consejo Nacional de Ciencia y Tecnología (CONACYT).

Durán Amaya, Ana Livier. 2006. Agave azul (Agave tequilana Weber) y el desarrollo rural sustentable: estudio de caso del municipio de Tonaya, Jalisco. Tesis de licenciatura, ingeniería en recursos naturales y agropecuarios. Universidad de Guadalajara.

García Hernández, José Luis. 1997. Estudio del maguey tequilero (Agave tequilana Weber) y su industrialización en la región centro del estado de Jalisco. Tesis de maestría. Universidad Autónoma Chapingo.

Gerritsen, Peter R.W. 2002. Diversity at Stake. A Farmers Perspective on Biodiversity and Conservation in Western Mexico. Wageningen: Wageningen University.

y Luis Manuel Martínez (editores). 2010. Agave azul, sociedad y medio ambiente.Guadalajara: CUCSUR, Universidad de Guadalajara.

y Jaime Morales H. (editores). 2007. Respuestas locales frente a la globalización. Productos regionales de la Costa Sur de Jalisco. Guadalajara: Universidad de Guadalajara, Instituto Tecnológico y de Estudios Superiores de Occidente (ITESO), Red de Alternativas Sustentables y Agropecuarias de Jalisco.

Lucie Leclert y Rodolfo González. 2007. Análisis de las destilerías de licor de agave en la sierra de Amula. Informe interno. Autlán: Universidad de Guadalajara.

Jesús Juan Rosales Adame, Arturo Moreno Hernández y Luis Manuel Martínez Rivera. 2006. Sistemas productivos y sustentabilidad rural en la Costa Sur de Jalisco en el occidente de México. Ponencia preparada para el $11^{0}$ Encuentro nacional sobre desarrollo regional en México, Mérida, Yucatán. 
Alma Lomelí J., y Claudia Ortiz A. 2005. Urbanización y problemática socioambiental en la Costa Sur de Jalisco, México: una aproximación. región y sociedad XVII (33): 107-132.

González, Marco Antonio. 2002. Blue Agave Producers in the Tequila Agro-industry in Jalisco, Mexico. The Beginning of Production Alliances in the Context of the End of Land Reform. Tesis de doctorado. University of Oxford.

Gutiérrez García, Octavio. 2005. Cuantificación de la erosión hídrica del suelo en el cultivo de agave azul (Agave tequilana Weber var. Azul), municipio de Autlán, Jalisco, México. Tesis de licenciatura, ingeniería en recursos naturales y agropecuarios. Universidad de Guadalajara.

Hostettler, Silvia. 2007. Land Use Changes and Transnational Migration: The Impact of Remittances in Western Mexico.Tesis de doctorado. Lausagne: École Polytechnique Féderale de Laqusanne.

IMECBIO. 2000. Programa de Desarrollo Regional Sustentable (PRODERS), región de la sierra de Manantlán (nivel regional). Informe técnico. Autlán: Secretaría de Medio Ambiente y Recursos Naturales /Universidad de Guadalajara.

Leclert, Lucie. 2007. Who Benefits from the "Denominación de Origen” Tequila? Tesis de maestría en sociología rural. Wageningen: Wageningen University.

Peter R.W. Gerritsen y Hielke van der Meulen. 2010. Agave azul: crisis cíclicas y las posibilidades para la planeación del cultivo en el estado de Jalisco. En Agave azul, sociedad y medio ambiente, editado por Peter R.W. Gerritsen y Luis Manuel Martínez, 19-42. Guadalajara: Universidad de Guadalajara.

Martínez Rivera, Luis Manuel, Peter R.W. Gerritsen, Jesús Juan Rosales A., Arturo Moreno H., Sarahy Contreras M., Arturo Solís M., Luis Eugenio Rivera C., Óscar Cárdenas H., Luis Ignacio Íñiguez 
D., Ramón Cuevas G., Carlos Palomera G., Edith García R., Ángel Aguirre G., y José Luis Olguín L. 2007. Implicaciones socioambientales de la expansión del cultivo de agave azul (1995-2002) en el municipio de Tonaya, Jalisco, México. En En lo ancestral hay futuro: del tequila, los mezcales y otros agaves, editado por P. Colunga, A. Larqué, L. Eguiarte y D. Zizumbo, 265-284. Mérida: CICY, CONACYT.

Morales Hernández, Jaime. 2004. Sociedades rurales y naturaleza. En busca de alternativas hacia la sustentabilidad. Guadalajara: ITESO / Universidad Iberamericana.

Nava L., Arturo, Arturo Moreno H., Peter R.W. Gerritsen y Jesús Juan Rosales A. 2006. El agave en Tonaya: tradición vs. globalización. Carta Económica Regional 19 (97): 3-9.

y Jorge Medina R. 2005. Evaluación del impacto económico del cultivo de agave (A. angustifolia Haw y A. tequilaza Weber) en el desarrollo productivo de Tonaya, Jalisco. Tesis de licenciatura, ingeniería en recursos naturales y agropecuarios. Universidad de Guadalajara.

O Connor, James. 2000. ¿Es posible el capitalismo sostenible? Revista Papeles de Población 24: 9-25.

Ploeg, Jan Douwe van der. 1994. Style of Farming. An Introductory Note on Concepts and Methodology. En Born from Within. Practice and Perspective of Endogenous Rural Development, editado por ídem., y A. Long, 7-30. Assen: Van Gorcum Publisher.

1992. The Reconstitution of Locality: Technology and Labour in Modern Agriculture. En Labour and Locality: Uneven Development and the Rural Labour Process, editado por T. Marsden, Robert Lowe y Sarah Whatmore, 19-43. Londres: David Fulton Publishers.

1990. Labor, Markets and Agricultural Production. Boulder, San Francisco y Oxford: Westview Press. 
Pretty, Jules N. 1995. Regenerating Agriculture: Policies and Practice for Sustainability and Self-reliance. Londres: Earthscan Publications Ltd.

Russell, Bernard. 1988. Research Methods in Cultural Anthropology. Newbury Park/London/New Delhi: SAGE Publications.

Schweighoefer, Nina. 2007. Hope and Disillusion, Unexpected and Underrepresented Implications of Tequila's Geographical Indication. Tesis de maestría en estudios de desarrollo internacional. Wageningen University.

Toledo, Víctor M. 2000. La paz en Chiapas. Ecología, luchas indígenas y modernidad alternativa. México: Ediciones Quinto Sol / Universidad Nacional Autónoma de México.

1995. Campesinidad, agroindustrialidad y sostenibilidad. Los fundamentos ecológicos e históricos del desarrollo rural. México: Grupo para el Desarrollo Sostenible de la Agricultura y los Recursos Naturales. Report No. 3.

Valenzuela Z., Ana G. 2003. El agave tequilera. Cultivo e industria de México. México, Madrid y Barcelona: Ediciones Mundi-Prensa.

Valenzuela, A. G., y P. R. W. Gerritsen. 2007. Memorias del primer seminario internacional de tequila: ambiente, cultura y sociedad. Autlán: CUCSUR, Universidad de Guadalajara/Signo Tequila, A.C.

Waters, Martin. 1995. Globalization. Londres y Nueva York: Routledge.

Zamora, D., Julián y Elsa Marbella Preciado B. 2003. Análisis socioambiental de la expansión del cultivo del agave azul (Agave tequilana Weber) en los municipios de Autlán de Navarro y Tuxcacuesco, Jalisco. Tesis de licenciatura, ingeniería en recursos naturales y agropecuarios. CUCSUR, Universidad de Guadalajara. 